\title{
ASYMPTOTICS OF DIFFERENTIAL SYSTEMS WITH DEVIATING ARGUMENTS
}

\author{
WILLIAM F. TRENCH
}

\begin{abstract}
Conditions are given which imply that a system $X^{\prime}(t)=$ $F[t, X(g(t))]$ has a solution with some components which approach given limits with specified orders of convergence as $t \rightarrow \infty$, while the other components have specified orders of magnitude. The integral smallness conditions on $F$ permit conditional convergence of some of the improper integrals that occur, and it is not required that $\lim _{t \rightarrow \infty} g(t)=\infty$.
\end{abstract}

1. Introduction. We consider the $n \times n$ system with deviating argument

$$
x_{i}^{\prime}(t)=f_{i}[t, X(g(t))], \quad 1 \leq i \leq n,
$$

where $X=\left(x_{1}, \ldots, x_{n}\right)$. The following assumption holds throughout.

ASSUMPTION A. $\psi_{1}, \ldots, \psi_{n}$ are positive and continuous on $[a, \infty)$, and $g$ is continuous on $(0, \infty)$, with

$$
\varliminf_{t \rightarrow \infty} g(t)=T_{0}>a \geq 0 .
$$

The sets $\mathcal{A}$ and $B$ form a partition of $\{1, \ldots, n\}$, and (a) if $i \in \mathcal{A}$, then either $\psi_{i}=1$ or $\psi_{i}(\tau)$ tends monotonically to zero as $\tau \rightarrow \infty$, (b) if $i \in B$, then $\psi_{i}(\tau)$ tends monotonically to infinity as $\tau \rightarrow \infty$. All quantities are real, and $C=\left(c_{1}, \ldots, c_{n}\right)$ is a given constant vector.

We say that $X=X(t)$ is a solution of $(1)$ on $\left[t_{0}, \infty\right)$ if $X$ is continuous for $t \geq \min \left\{t_{0}, \hat{t}_{0}\right\}$, where $\hat{t}_{0}=\inf \left\{g(s) \mid s \geq t_{0}\right\}$, and satisfies (1) for $t>t_{0}$. We give conditions implying that if $t_{0}$ is sufficiently large, then (1) has a solution $\bar{X}$ on $\left[t_{0}, \infty\right)$, such that

$$
\begin{cases}\bar{x}_{i}(t)=c_{i}+O\left(\psi_{i}(t)\right), & i \in \mathcal{A}, \\ \bar{x}_{i}\left(t_{0}\right)=c_{i} \quad \text { and } \quad \bar{x}_{i}(t)=O\left(\psi_{i}(t)\right), & i \in \mathcal{B}, \quad(t \rightarrow \infty),\end{cases}
$$

where " $O$ " may be replaced by " $O$ " in some cases.

Our integral smallness conditions on $f_{1}, \ldots, f_{n}$ permit conditional convergence of some of the improper integrals that occur. This continues a theme developed in [5] with $g(t)=t$ and $A=\{1, \ldots, n\}$. (For earlier results on systems of ordinary differential equations subject to possibly conditional integral smallness conditions, see Hallam $[\mathbf{1}, \mathbf{2}]$.)

Although much has been written on the asymptotic properties of systems of ordinary differential equations, and on scalar equations with deviating arguments, relatively little has been published on systems with deviating arguments. (For examples, see Kitamura and Kusano [3, 4].)

Received by the editors August 16, 1983. Presented to the Society, April 1983.

1980 Mathematics Subject Classification. Primary 34E10, 34C99.

(C) 1984 American Mathematical Society $0002-9939 / 84 \$ 1.00+\$ .25$ per page 
2. The main theorem. If $h_{1}, \ldots, h_{n}$ are locally integrable on $[\tau, \infty)$ and $T \geq \tau \geq a$, let

$$
\begin{array}{ll}
I\left(t ; h_{i}\right)=\left|\int_{t}^{\infty} h_{i}(s) d s\right|, \quad i \in \mathcal{A} \\
I\left(t ; h_{i}\right)=\left|\int_{T}^{t} h_{i}(s) d s\right|, \quad i \in B
\end{array}
$$

We will state assumptions below of the form

$$
\varlimsup_{t \rightarrow \infty}\left(\psi_{i}(t)\right)^{-1} I\left(t ; h_{i}\right)=\alpha_{i}<\infty, \quad 1 \leq i \leq n .
$$

In all cases it is implicitly assumed that $\int^{\infty} h_{i}(s) d s$ converges for $i \in \mathcal{A}$, perhaps conditionally if $h_{i}$ is not eventually sign constant. Obviously, $\alpha_{i}=0$ if $i \in \mathcal{A}$ and $\psi_{i}=1$.

REMARK 1. The constants $\alpha_{1}, \ldots, \alpha_{n}$ in (4) are independent of $T$. This is obvious if $i \in A$; if $i \in B$, it follows from the assumption that $\lim _{t \rightarrow \infty} \psi_{i}(t)=\infty$. If $\varepsilon>0$ and $i \in B$, then (4) implies that there is a $t_{0} \geq T$ such that

$$
\left|\int_{t_{0}}^{t} h_{i}(s) d s\right| \leq 2\left(\alpha_{i}+\varepsilon\right) \psi_{i}(t), \quad t \geq t_{0},
$$

and the two may be dropped if $h_{i}$ is sign constant on $[T, \infty)$.

THEOREM 1. Suppose there are constants $T, M_{1}, \ldots, M_{n}$ such that

$$
a<T<T_{0} ; \quad M_{i}>0, \quad 1 \leq i \leq n,
$$

$g(t) \geq a(t \geq T)$, and $f_{1}, \ldots, f_{n}$ are continuous and satisfy

$$
\left|f_{i}(t, Y)-f_{i}(t, C)\right| \leq w_{i}\left(t,\left|y_{1}-c_{1}\right|, \ldots,\left|y_{n}-c_{n}\right|\right), \quad 1 \leq i \leq n,
$$

on the set

$$
\Omega=\left\{(t, Y)|t \geq T ;| y_{j}-c_{j} \mid \leq M_{j} \psi_{j}(g(t)), 1 \leq j \leq n\right\},
$$

where $w_{i}=w_{i}\left(t, \xi_{1}, \ldots, \xi_{n}\right)$ is continuous for $t \geq T, \xi_{j} \geq 0(1 \leq j \leq n)$, and nondecreasing in $\xi_{1}, \ldots, \xi_{n}$. Define

$$
\sigma_{i}(t)=w_{i}\left(t, M_{1} \psi_{1}(g(t)), \ldots, M_{n} \psi_{n}(g(t))\right), \quad t \geq T .
$$

Suppose also that

$$
\varlimsup_{t \rightarrow \infty}\left(\psi_{i}(t)\right)^{-1} I\left(t ; f_{i}(\cdot ; C)\right)=A_{i}<\infty, \quad 1 \leq i \leq n,
$$

and

$$
\varlimsup_{t \rightarrow \infty}\left(M_{i} \psi_{i}(t)\right)^{-1} I\left(t ; \sigma_{i}\right)=\theta_{i}<1, \quad 1 \leq i \leq n,
$$

where

$$
M_{i}>A_{i} /\left(1-\theta_{i}\right), \quad i \in \mathcal{A} ; \quad M_{i}>2 A_{i} /\left(1-\theta_{i}\right), \quad i \in B .
$$

Then, if $t_{0}$ is sufficiently large, (1) has a solution $\bar{X}$ on $\left[t_{0}, \infty\right)$ such that

$$
\left|\bar{x}_{i}(t)-c_{i}\right| \leq M_{i} \psi_{i}(t), \quad t \geq t_{0}, 1 \leq i \leq n ; \quad \bar{x}_{i}\left(t_{0}\right)=c_{i}, \quad i \in B
$$

and

$$
\varlimsup_{t \rightarrow \infty}\left(\psi_{i}(t)\right)^{-1}\left|\bar{x}_{i}(t)-c_{i}\right| \leq A_{i}+M \theta_{i}, \quad 1 \leq i \leq n
$$


Proof. Choose $t_{0} \geq T$ so that

$$
g(t) \geq T, \quad t \geq t_{0}
$$

(see (2) and (5)), and so that the functions

$$
\mu_{i}(t)= \begin{cases}\int_{t}^{\infty} \sigma_{i}(s) d s+\left|\int_{t}^{\infty} f_{i}(s, C) d s\right|, & i \in \mathcal{A}, \\ \int_{t_{0}}^{t} \sigma_{i}(s) d s+\left|\int_{t_{0}}^{t} f_{i}(s, C) d s\right|, & i \in B\end{cases}
$$

satisfy the inequalities

$$
\mu_{i}(t) \leq M_{i} \psi_{i}(t), \quad t \geq t_{0}
$$

This is possible because of (9)-(11). (See Remark 1.) Let $C_{T}$ be the space of continuous $n$-vector functions on $[T, \infty)$ with the topology of uniform convergence on finite intervals, and let

$$
\mathcal{H}=\left\{X \in C_{T}|| x_{i}(\tau)-c_{i} \mid \leq M_{i} \psi_{i}(\tau), \tau \geq T, 1 \leq i \leq n\right\} .
$$

Define the transformation $\hat{X}=\tau X$ by

$$
\begin{aligned}
& \hat{x}_{i}(t)= \begin{cases}c_{i}-\int_{t}^{\infty} f_{i}[s, X(g(s))] d s, & t \geq t_{0}, \\
c_{i}-\int_{t_{0}}^{\infty} f_{i}[s, X(g(s))] d s, & T \leq t \leq t_{0},\end{cases} \\
& \hat{x}_{i}(t)= \begin{cases}c_{i}+\int_{t_{0}}^{t} f_{i}[s, X(g(s))] d s, & t \geq t_{0}, \\
c_{i}, & T \leq t \leq t_{0},\end{cases}
\end{aligned}
$$

We will use the Schauder-Tychonoff theorem to show that $\bar{X}=\tau \bar{X}$ for some $\bar{X}$ in $\mathcal{K}$, and then verify that $\bar{X}$ has the stated properties.

Clearly $\mathcal{H}$ is a closed convex subset of the Fréchet space $C_{T}$. If $X \in \mathcal{H}$, then (14) and (17) imply that $(t, X(g(t))) \in \Omega$ for $t \geq t_{0}$, so the functions $h_{i}(t)=f_{i}[t, X(g(t))]$ are continuous on $\left[t_{0}, \infty\right)$, and (6), (7), and the monotonicity of $w_{i}$ imply that

$$
\left|f_{i}[t, X(g(t))]-f_{i}(t, C)\right| \leq \sigma_{i}(t), \quad t \geq t_{0}, 1 \leq i \leq n, X \in \nVdash .
$$

By writing

$$
\int f_{i}[s, X(g(s))] d s=\int\left(f_{i}[s, X(g(s))]-f_{i}(s, C)\right) d s+\int f_{i}(s, C) d s,
$$

it is easily seen from (15), (18), (19), and (20) that

$$
\left|\hat{x}_{i}(t)-c_{i}\right| \leq \mu_{i}(t) \leq M_{i} \psi_{i}(t), \quad t \geq t_{0}, 1 \leq i \leq n,
$$

where the second inequality comes from (16). Since $\psi_{i}(t) \geq \psi_{i}\left(t_{0}\right)$ if $T \leq t \leq t_{0}$ and $i \in A,(21)$ and the definition of $\hat{X}$ on this interval imply that $\hat{X} \in \mathcal{H} ;$ thus, $\tau$ maps $H$ into itself.

If $X$ and $X_{j}$ are in $\mathcal{H}$, then

$$
\left|\hat{x}_{i j}(t)-\hat{x}_{i}(t)\right| \leq \int_{t_{0}}^{\infty}\left|f_{i}\left[s, X_{j}(g(s))\right]-f_{i}[s, X(g(s))]\right| d s, \quad t \geq T
$$


if $i \in A($ see (18)), and

$$
\left|\hat{x}_{i j}(t)-\hat{x}_{i}(t)\right| \leq \int_{t_{0}}^{t_{1}}\left|f_{i}\left[s, X_{j}(g(s))\right]-f_{i}[s, X(g(s))]\right| d s, \quad T \leq t \leq t_{1},
$$

if $i \in B$ (see (19)). From (20), $2 \sigma_{i}(s)$ majorizes the integrands in (22) and (23); hence, (10) and Lebesgue's dominated convergence theorem imply that if $\lim _{j \rightarrow \infty} X_{j}(t)=X(t)(t \geq T)$, then $\lim _{j \rightarrow \infty} \hat{x}_{i j}(t)=\hat{x}_{i}(t)(t \geq T)$ with uniform convergence on $[T, \infty)$ if $i \in \mathcal{A}$ and on $\left[T, t_{1}\right]$ for every $t_{1}>T$ if $i \in B$. Therefore, $T$ is continuous on $\forall$.

If $X \in \mathcal{H}$, then (18) and (19) imply that $x_{i}^{\prime}=0$ on $\left[T, t_{0}\right]$, and

$$
\left|x_{i}^{\prime}(t)\right|=\left|f_{i}[t, X(g(t))]\right| \leq \sigma_{i}(t)+\left|f_{i}(t, C)\right|, \quad t \geq t_{0}, 1 \leq i \leq n
$$

(see $(20))$. Hence, the family $T(\not{H})$ is equicontinuous on compact subsets of $[T, \infty)$. Since $\tau(\mathscr{H}) \subset \mathcal{H},(17)$ implies that $\tau(\mathscr{H})$ is uniformly bounded on such subsets. Therefore, the Arzela-Ascoli theorem implies that $\tau(\not{H})$ has compact closure.

The Schauder-Tychonov theorem now implies that $\bar{X}=\tau \bar{X}$ for some $\bar{X}$ in $\nLeftarrow$. Setting $x_{i}=\hat{x}_{i}=\bar{x}_{i}$ in (18) and (19) shows that $\bar{X}$ is a solution of (1) on $\left[t_{0}, \infty\right)$ and $\bar{x}_{i}\left(t_{0}\right)=c_{i}$ if $i \in B$. For (13), we observe that $\left|\bar{x}_{i}(t)-c_{i}\right| \leq \mu_{i}(t)$, because of (21) with $\hat{x}_{i}=\bar{x}_{i}$, and that $\varlimsup_{t \rightarrow \infty}\left(\psi_{i}(t)\right)^{-1} \mu_{i}(t) \leq A_{i}+M \theta_{i}$, because of (9), (10), and (15). This completes the proof.

3. An application to a linear system. The linear system

$$
x_{i}^{\prime}(t)=\sum_{j=1}^{n} a_{i j}(t) x_{j}(g(t))+h_{i}(t), \quad 1 \leq i \leq n,
$$

is of the form (1), with

$$
f_{i}(t, Y)=\sum_{j=1}^{n} a_{i j}(t) y_{j}+h_{i}(t)
$$

Let

$$
\lambda_{i}(t)=\sum_{j=1}^{n}\left|a_{i j}(t)\right| \psi_{j}(g(t))
$$

THEOREM 2. Suppose that $a_{i j}, h_{i} \in C(0, \infty)$ and (9) holds, with $f_{i}$ as in (25). Suppose also that

$$
\varlimsup_{t \rightarrow \infty}\left(\psi_{i}(t)\right)^{-1} I\left(t ; \lambda_{i}\right)=\theta_{i}<1, \quad 1 \leq i \leq n .
$$

Then (24) has a solution $\bar{X}$ on $\left[t_{0}, \infty\right)$ which satisfies (3), provided $t_{0}$ is sufficiently large; moreover, if $A_{i}=\theta_{i}=0$ for some $i$, then " $O$ " may be replaced by " 0 " in (3) for that $i$.

PROOF. In this case (6) holds on $(0, \infty) \times R^{n}$, with

$$
w_{i}\left(t, \xi_{1}, \ldots, \xi_{n}\right)=\sum_{j=1}^{n}\left|a_{i j}(t)\right| \xi_{j} .
$$


Let $T$ be in $\left(a, T_{0}\right)$ and $M_{1}=\cdots=M_{n}=M$, where $M$ is chosen to satisfy (11); then (8) becomes $\sigma_{i}(t)=M \lambda_{i}(t)$ (see (26)), so (27) implies (10). Now Theorem 1 implies the conclusion.

EXAMPLE. Consider the system

$$
\left[\begin{array}{l}
x_{1}^{\prime}(t) \\
x_{2}^{\prime}(t)
\end{array}\right]=\left[\begin{array}{cc}
2 t^{-2} \sin t & t^{-1} \\
t^{-3} & t^{-3} \sin t
\end{array}\right]\left[\begin{array}{l}
x_{1}\left(t^{\nu}\right) \\
x_{2}\left(t^{\nu}\right)
\end{array}\right]+\left[\begin{array}{c}
6 t^{-1} \\
-2 t^{-3}
\end{array}\right],
$$

where $\nu>0$. Here $\lambda_{1}$ and $\lambda_{2}$ in (26) are

$$
\begin{aligned}
& \lambda_{1}(t)=2 t^{-2}|\sin t| \psi_{1}\left(t^{\nu}\right)+t^{-1} \psi_{2}\left(t^{\nu}\right), \\
& \lambda_{2}(t)=t^{-3} \psi_{1}\left(t^{\nu}\right)+t^{-3}|\sin t| \psi_{2}\left(t^{\nu}\right) .
\end{aligned}
$$

If $C=\left(c_{1}, c_{2}\right)$, then

$$
\int_{T}^{t} f_{1}(s, C) d s=\left(c_{2}+6\right) \log t+O(1)
$$

and

$$
\int_{t}^{\infty} f_{1}(s, C) d s=O\left(t^{-2}\right) \quad \text { if } c_{2}=-6
$$

In any case,

$$
\int_{t}^{\infty} f_{2}(s, C) d s=\frac{\left(c_{1}-2\right) t^{-2}}{2}+O\left(t^{-3}\right) .
$$

We first apply Theorem 2 with $A=\{2\}$ and $B=\{1\}$. If $\psi_{1}(t)=\log t$, then (31) implies (9) for $i=1$ and (30) implies that

$$
\int_{t}^{\infty} \lambda_{2}(s) d s=\frac{\nu}{2} t^{-2} \log t+O\left(t^{-2}\right)
$$

if $\psi_{2}$ is any nonincreasing function. Therefore, (27) holds for $i=2$ if $\nu<2$ and $\psi_{2}(t)=t^{-2} \log t$, or if $\nu \geq 2$ and $\psi_{2}(t)=t^{-2+\alpha} \log t$, where $\alpha$ is any positive number; moreover, $\theta_{2}=0$ in the second case. Since (27) holds for $i=1$ with $\theta_{1}=0$ in either case (if we assume, without loss of generality, that $\alpha<2$ ), Theorem 2 implies that if $t_{0}$ is sufficiently large, then (28) has a solution $\bar{X}$ on $\left[t_{0}, \infty\right)$ such that $\bar{x}_{1}\left(t_{0}\right)=c_{1}, \bar{x}_{1}(t)=O(\log t)$, and $\bar{x}_{2}(t)=c_{2}+O\left(t^{-2} \log t\right)$ if $\nu<2$, or $\bar{x}_{2}(t)=c_{2}+o\left(t^{-2+\alpha} \log t\right)$ if $\nu \geq 2$.

If $c_{2}=-6$, we can apply Theorem 2 with $\alpha=\{1,2\}$ and $B$ empty. From (32) and (33), it seems reasonable to let $\psi_{1}(t)=t^{-a}$ and $\psi_{2}(t)=t^{-b}$, with $a$ and $b$ as large as possible. If $c_{1} \neq 2$, then (32) and (33) dictate that $a \leq 2$ and $b \leq 2$. If $\lambda_{1}$ and $\lambda_{2}$ in (29) and (30) are to satisfy (27), then we must have $\nu a+1 \geq a, \nu b \geq a, \nu a+2 \geq b$, and $\nu b+2 \geq b$. If $\nu>1$, let $a=b=2$; then (27) holds with $\theta_{1}=\theta_{2}=0$, and so (28) has a solution $\bar{X}$ such that $\bar{x}_{1}(t)=c_{1}+O\left(t^{-2}\right)$ and $\bar{x}_{2}(t)=-6+O\left(t^{-2}\right)$. If $\nu \leq 1$, let $a=2 \nu$ and $b=2$; then (27) holds with $\theta_{1}=\frac{1}{2} \nu$ and $\theta_{2}=0$, so (28) has a solution $\bar{X}$ such that $\bar{x}_{1}(t)=c_{1}+O\left(t^{-2 \nu}\right)$ and $\bar{x}_{2}(t)=-6+O\left(t^{-2}\right)$ if $1 / 2<\nu \leq 1$. If $0<\nu \leq 1 / 2$ and $\alpha>0$, then (28) has a solution $\bar{X}$ such that $\bar{x}_{1}(t)=c_{1}+o\left(t^{-2 \nu+\alpha}\right)$ and $\bar{x}_{2}(t)=-6+O\left(t^{-2}\right)$. Sharper estimates can be obtained for the case where $C=(2,-6)$, but we omit the details. 


\section{REFERENCES}

1. T. G. Hallam, Asymptotic integration of second order differential equations with integrable coefficients, SIAM J. Appl. Math. 19 (1970), 430-439.

2. _ Asymptotic integration of a nonhomogeneous differential equation with integrable coefficients, Czechoslovak Math. J. 21 (1971), 661-671.

3. Y. Kitamura and T. Kusano, On the oscillation of a class of nonlinear differential systems with deviating argument, J. Math. Anal. Appl. 66 (1978), 20-36.

4. __ Oscillation and a class of nonlinear differential systems with general deviating arguments, Nonlinear Anal. 2 (1978), 537-551.

5. W. F. Trench, Systems of differential equations subject to mild integral conditions, Proc. Amer. Math. Soc. 87 (1983), 263-270.

Department of MATHEMatics and COMPUTER SCIENCE, DREXel University, Philadelphia, Pennsylvania 19104 